\title{
Some Comments on the Non-Self-Dual Nahm Equations
}

\author{
E. Corrigan *, P. R. Wainwright, and S. M. J. Wilson \\ Department of Mathematical Sciences, University of Durham, Durham DH1 3LE, England
}

\begin{abstract}
Some empirical results on the cubic algebra $2 a_{i}=\sum_{j}\left[a_{j},\left[a_{j}, a_{i}\right]\right]$ are presented. The algebra is satisfied at the residue of any pole in a solution to Nahm's non-self-dual equations.
\end{abstract}

\section{Introduction}

Recently, there has been some interest in Yang-Mills equations in one dimension, reduced from four by insisting that all fields be independent of three of the variables. On the one hand, some work has been done on the mechanical system which results when the four dimensional theory is defined in Minkowski space and the reduction is simply to delete all dependence on the cartesian spatial variables $\mathbf{x}$ [1]. This mechanical system has been shown to be essentially chaotic by Nikolaevskii and Schur and by Savviddy [2]. On the other hand, if we take the Euclidean version of the four dimensional theory and reduce it by deleting the dependence on any three of the cartesian coordinates, we obtain a set of equations, differing from the previous set only by a single sign but which is interesting in a very different context. In this case, certain solutions to the self-dual equations, which imply the second order equations, of course, have a remarkable role to play. They are intimately related via the ADHMN [3] (Atiyah-Drinfeld-Hitchin-Manin$\mathrm{Nahm}$ ) construction to static monopole solutions to Yang-Mills-Higgs theories in four dimensions in the Bogomol'nyi-Prasad-Sommerfield limit [4]. Indeed, the ADHMN construction may be viewed [5] as an equivalence between two sets of self-dual equations (with appropriate boundary conditions), one as described above in one dimension, the other in three dimensions (reduced from a Euclidean four dimensional theory by deleting dependence on a single variable); the latter corresponding to the equations satisfied by the vector potential and Higgs' field in the BPS limit.

It is known that the SU(2) Yang-Mills-Higgs theory has solutions of finite energy which do not satisfy the first order self-dual equations but are, nevertheless, solutions to the second order field equations [6]. Taubes' existence proof of this

* Sir Derman Christopherson Foundation Fellow 
fact is non-constructive and an outstanding problem is to try to find an approach to these extra solutions, which are to be regarded conceivably as carefully balanced monopole-antimonopole composites, still static but unstable.

An idea which we should like to explore is the possibility that the Nahm transformation between two sets of self-dual equations actually extends also to some sort of transformation between non-self-dual (and possibly even non-static) solutions. There are enormous technical difficulties in attempting to verify such an idea and we shall not be able to pursue it very far here. However, it seems a plausible possibility and suggests that it would be wothwhile cataloguing the properties of non-self-dual solutions to the one dimensional theory. This is already a formidable task. In this article our aim is to describe some modest discoveries that we consider to be quite interesting. In particular, we shall demonstrate the existence of simple solutions to the second order equations which interpolate between self-dual and antidual solutions, and explore some of their properties.

\section{One Dimensional Yang-Mills Equations}

Starting with the full Yang-Mills equations in four Euclidean dimensions,

$$
D_{\mu} T_{\mu \nu}=0, \quad \mu, v=1, \ldots, 4,
$$

where

$$
\begin{aligned}
T_{\mu \nu} & =\partial_{\mu} T_{\nu}-\partial_{\nu} T_{\mu}-i\left[T_{\mu}, T_{\nu}\right], \\
T_{\mu} & =T_{\mu}^{\dagger}, \\
D_{\mu} & =\partial_{\mu}-i\left[T_{\mu},\right],
\end{aligned}
$$

and demanding all fields be independent of three of the variables, say $x_{1}, x_{2}, x_{3}$, we obtain (setting $x_{4}=z$ ),

$$
\begin{gathered}
\frac{d^{2} T_{i}}{d z^{2}}-\left[T_{j},\left[T_{j}, T_{i}\right]\right]=0, \quad i, j=1,2,3, \\
{\left[T_{j}, \frac{d T_{j}}{d z}\right]=0,}
\end{gathered}
$$

in the gauge $T_{4}=0$. We shall further suppose the vector potential $T_{\mu}$ to be traceless, corresponding to an $\mathrm{SU}(N)$ gauge field.

The self-dual equations corresponding to (3), (4) are

$$
\frac{d T_{i}}{d z}= \pm i \varepsilon_{i j k} T_{j} T_{k}
$$

It is easy to check directly that Eq. (5) implies Eqs. (3) and (4).

There is an obvious conserved quantity associated with Eqs. (3),

$$
\begin{aligned}
E & =\operatorname{tr}\left(\frac{d T}{d z}\right)^{2}+\frac{1}{2} \operatorname{tr}\left(\left[T_{i}, T_{j}\right]\left[T_{i}, T_{j}\right]\right) \\
& =\operatorname{tr}\left\{\left(\frac{d T_{k}}{d z}-i \varepsilon_{k l m} T_{l} T_{m}\right)\left(\frac{d T_{k}}{d z}+i \varepsilon_{k n p} T_{n} T_{p}\right)\right\},
\end{aligned}
$$


which is not positive for all field configurations. From the second expression (7) it clearly vanishes for a self-dual or an anti-dual solution. For the analogous equations to (3) for a theory reduced from Minkowski space, in which $z$ plays the role of time, one of the two terms in Eq. (3) changes sign and the conserved quantity $E$ is a sum of squares. It corresponds to the energy of the mechanical system.

In order to construct monopoles in an SU(2) Yang-Mills-Higgs theory the starting point is the set of solutions to Eq. (5) defined in the interval $|z| \leqq \frac{1}{2}$ and regular except for simple poles at $z= \pm \frac{1}{2}$. Clearly, the residue of such a simple pole

$$
T_{i} \sim \frac{\alpha_{i}}{z+\frac{1}{2}}
$$

must satisfy

$$
\alpha_{i}=\mp i \varepsilon_{i j k} \alpha_{j} \alpha_{k} .
$$

In other words, either $\alpha_{i}$ or $-\alpha_{i}$ must satisfy an SU(2) Lie algebra. For the monopoles alluded to above $\pm \alpha_{i}$ must be an irreducible representation of the algebra at both ends of the interval $|z| \leqq \frac{1}{2}$.

From the point of view of the self-dual equations (5) the simple pole is a fundamental solution in the sense that it is the unique functional form for which the ansatz

$$
T_{i}=a(z) \alpha_{i}, \quad i=1,2,3,
$$

where the $\alpha_{i}$ are constant matrices, can work.

For the second order equations the ansatz (9) and a convenient choice of scaling leads to the pair of equations

$$
\begin{gathered}
2 \alpha_{i}=\sum_{j=1}^{3}\left[\alpha_{j},\left[\alpha_{j}, \alpha_{i}\right]\right], \\
\frac{d^{2} a}{d z^{2}}=2 a^{3} .
\end{gathered}
$$

Equation (11) is easily solved in terms of elliptic functions [7] and its general solution is

$$
a(z)=\sigma \frac{\operatorname{sn} \sigma\left(z-z_{0}\right) d n \sigma\left(z-z_{0}\right)}{\operatorname{cn} \sigma\left(z-z_{0}\right)},
$$

with the elliptic functions all defined for $k=1 / \sqrt{2}$, the symmetric point. The constants $\sigma, z_{0}$ are arbitrary. To arrange poles at $z= \pm \frac{1}{2}$ with $a(z)$ regular within $|z| \leqq \frac{1}{2}$ we would choose $z_{0}=0$ and $\sigma=2 K(1 / \sqrt{2})$, where $K(k)$ is the complete elliptic integral of the first kind. A single pole may be arranged as a special limit by sending $\sigma$ to zero.

For these solutions the conserved quantity simplifies and is given by

$$
E=\sigma^{4} \operatorname{tr}\left(\alpha_{i} \alpha_{i}\right)
$$

It is clearly positive except for the single pole, where it vanishes.

Equation (10) is not so easily analysed and it is to this that we wish to concentrate our attention. We note it must be satisfied by the residue of any pole of 
$T_{i}(z)$ even if the solution to Eqs. (3), (4) is not of the simplest type just discussed. Clearly, if $\pm \alpha_{i}$ satisfies the SU(2) Lie algebra, then Eq. (10) is satisfied identically. However, we are interested in discovering other more general solutions than these. For brevity we shall refer to (10) as a "cubic algebra".

\section{Exploring the Cubic Algebra}

Clearly, any solution to the cubic algebra is part of a continuous family of solutions obtained as an orbit under the product group $\mathrm{SO}(3) \times \mathrm{SU}(N)$. The action of this group on $\alpha_{i}$ is given as follows:

$$
\alpha_{i} \rightarrow O_{i j} U^{\dagger} \alpha_{j} U, \quad O \in \mathrm{SO}(3), \quad U \in \mathrm{SU}(N) .
$$

The cubic algebra is invariant under this set of transformations because of the orthogonality of $O$ and unitarity of $U$.

There are many invariants that may be constructed from the $\alpha$ 's, but two important ones which will allow us to distinguish all the solutions we shall consider are $q, t$ given respectively by

$$
\begin{gathered}
q=\operatorname{tr}\left(\alpha_{i} \alpha_{i}\right), \\
t=-i \varepsilon_{i j k} \operatorname{tr}\left(\alpha_{i} \alpha_{j} \alpha_{k}\right) .
\end{gathered}
$$

We note the value of $t$ is bounded by $q$

$$
q \geqq|t|
$$

whenever the $\alpha$ 's satisfy the cubic algebra. Moreover, $q= \pm t$ if and only if $\pm \alpha_{i}$ represents the SU(2) algebra. To see (17) we note the inequality,

$$
0 \leqq \operatorname{tr}\left\{\left(\alpha_{i} \pm i \varepsilon_{i j k} \alpha_{j} \alpha_{k}\right)\left(\alpha_{i} \pm i \varepsilon_{i l m} \alpha_{l} \alpha_{m}\right)\right\}=2(q \mp t) \text {, }
$$

which follows directly from the cubic algebra.

It is convenient sometimes to write the cubic algebra in a complex form, setting

$$
\alpha_{ \pm}=\alpha_{1} \pm i \alpha_{2}, \quad \alpha_{0}=\alpha_{3},
$$

in which case the algebra reads

$$
\begin{aligned}
4 \alpha_{0} & =\left[\alpha_{+},\left[\alpha_{-}, \alpha_{0}\right]\right]+\left[\alpha_{-},\left[\alpha_{+}, \alpha_{0}\right]\right], \\
4 \alpha_{+} & =\left[\alpha_{+},\left[\alpha_{-}, \alpha_{+}\right]\right]+2\left[\alpha_{0},\left[\alpha_{0}, \alpha_{+}\right]\right] .
\end{aligned}
$$

A natural nonlinear mapping of the $\alpha$ 's which we shall consider is to replace the three $\alpha$ 's by the commutators of each pair. Thus

$$
\alpha \rightarrow \tilde{\alpha}(\alpha) \text {, }
$$

where

$$
\tilde{\alpha}_{0}=\frac{1}{2}\left[\alpha_{+}, \alpha_{-}\right], \quad \tilde{\alpha}_{ \pm}= \pm\left[\alpha_{0}, \alpha_{ \pm}\right] .
$$

Obviously, if $\alpha$ represents the SU(2) Lie algebra then it is a fixed point of this mapping. We shall find that other solutions also have special distinguished properties under (20), though it is not always the case that $\tilde{\alpha}$ solves the cubic algebras when $\alpha$ does. We also note that the commutator mapping preserves the 
quadratic invariant $q$ when $\alpha$ solves the cubic algebra. It does not, however, preserve the cubic invariant $t$ except in special cases.

We now turn to a detailed study of the two and three dimensional cases.

$N=2$. In this case there are just three inequivalent types of solution. The matrices $\pm \alpha$ either represent $\mathrm{SU}(2)$ or are equivalent to

$$
\alpha=\frac{1}{\sqrt{2}}\left(\sigma_{1}, \sigma_{2}, 0\right) \text {. }
$$

This is easy to prove. We may write each of the matrices $\alpha_{i}$ as a real linear combination of the Pauli $\sigma$-matrices

$$
\alpha_{i}=A_{i j} \sigma_{j} / 2
$$

Then the cubic algebra reads

$$
A A^{T} A=A\left(\operatorname{tr}\left(A^{T} A\right)-2\right),
$$

and each of the matrices $A A^{T}$ and $A^{T} A$ satisfies

$$
x^{2}=x(\operatorname{tr} x-2) .
$$

Thus, either $x$ is invertible, in which case it must be the identity, or it has one zero eigenvalue. Otherwise, $x=0$ is the only other possibility. Up to the equivalences (14) $A$ can be chosen to be either ( \pm ) the identity or to be diagonal with entries $(\sqrt{2}, \sqrt{2}, 0)$.

For the solution (22) the invariant $t$, Eq. (16), vanishes. This solution is singular in the sense that applying the commutator mapping (20) to it fails to produce a new solution, but applying it twice yields zero, the trivial solution. We can also think of this solution as a "twisted" linear combination of the two self-dual solutions, $\alpha^{( \pm)}= \pm \frac{1}{2} \sigma$, in the following way. It is equivalent to $\alpha\left(\frac{\pi}{2}\right)$ when

$$
\alpha(\theta)=\boldsymbol{\alpha}^{(+)}+R(\theta) \boldsymbol{\alpha}^{(-)},
$$

where $R(\theta)$ is an $\mathrm{SO}(3)$ rotation about the third axis through an angle $\theta$.

None of the two dimensional solutions belongs to an orbit depending on the full six parameters of $\mathrm{SO}(3) \times \mathrm{SU}(2)$. Indeed, the self-dual solutions $\boldsymbol{\alpha}^{( \pm)}$have a "spherical" symmetry in the sense that any $\mathrm{SO}(3)$ transformation can be compensated by a corresponding SU(2) transformation; their orbit is described by just three parameters. The singular solution (22) has a similar "cylindrical" symmetry and its orbit depends on only five parameters.

$N=3$. This case is already too difficult to analyse completely at present. Clearly, there are two inequivalent ways of embedding the SU(2) Lie algebra in the Lie algebra of $\mathrm{SU}(3)$. One of these, the maximal embedding, organizes the eight generators of $\mathrm{SU}(3)$ into two multiplets, of dimension three (the embedding) and five. The other organizes the generators into a triplet (the embedding), two doublets and a singlet. The latter is what one might call the Gell-Mann isospin embedding. For each of these there is a pair of dual solutions and a singular solution, just as for the case $N=2$ discussed above. They are distinguished by the invariants $(q, t)$ which have the values $(3 / 2, \pm 3 / 2),(6, \pm 6)$ for the two embeddings, and $(2.0)$. (8.0) for the corresponding singular solutions. 
However, these solutions do not exhaust the possibilities. There are others. In particular, we have discovered a continuous family of inequivalent solutions interpolating the $( \pm)$ maximal embeddings. It was originally found during a computer search (for special cases of the parameter) but can be understood in terms of the maximal embedding in the following way.

It is convenient to choose a Chevalley basis for the generators of the $\mathrm{SU}(3)$ algebra in terms of a pair of simple roots $\alpha, \beta$ satisfying

$$
\alpha^{2}=\beta^{2}=1, \quad \alpha \cdot \beta=-\frac{1}{2}
$$

and the conventions [8]:

$$
\begin{aligned}
{\left[\mathbf{H}, E_{r}\right] } & =\mathbf{r} E_{r}, \\
{\left[E_{r}, E_{-r}\right] } & =2 \mathbf{r} \cdot \mathbf{H}, \\
{\left[E_{ \pm \alpha}, E_{ \pm \beta}\right] } & = \pm E_{ \pm(\alpha+\beta)}, \\
{\left[E_{-\beta}, E_{\alpha+\beta}\right] } & =-E_{\alpha}, \\
{\left[E_{-\alpha}, E_{\alpha+\beta}\right] } & =E_{\beta}, \\
E_{r}^{+} & =E_{-r}, \quad \text { if } r \text { is any root },
\end{aligned}
$$

Using these there are many equivalent ways of choosing a maximal embedding. However, a particularly nice one is to write

$$
\begin{aligned}
J_{+} & =\sqrt{2}\left(E_{\alpha}+E_{\beta}\right)=J_{-}^{+}, \\
J_{0} & =2(\boldsymbol{\alpha}+\boldsymbol{\beta}) \cdot \mathbf{H} .
\end{aligned}
$$

With respect to this choice the other five generators are an $\mathrm{SO}(3)$ quintuplet, which we may write as

$$
\begin{aligned}
& Q_{2}=E_{\alpha+\beta}=Q_{-2}^{\dagger}, \\
& Q_{1}=-\frac{1}{\sqrt{2}}\left(E_{\alpha}-E_{\beta}\right)=-Q_{-1}^{\dagger}, \\
& Q_{0}=\frac{2}{\sqrt{6}}(\boldsymbol{\alpha}-\boldsymbol{\beta}) \cdot \mathbf{H},
\end{aligned}
$$

where the normalizations have been chosen so that the standard commutation relations

$$
\begin{aligned}
{\left[J_{0}, Q_{m}\right] } & =m Q_{m}, \\
{\left[J_{ \pm}, Q_{m}\right] } & =\sqrt{(3 \pm m)(2 \mp m) Q_{m \pm 1}}
\end{aligned}
$$

are obeyed. In addition, we may also note that amongst themselves the only nonzero commutators of the $Q$ 's are

$$
\begin{array}{ll}
{\left[Q_{2}, Q_{-2}\right]=J_{0},} & {\left[Q_{1}, Q_{-1}\right]=-\frac{1}{2} J_{0},} \\
{\left[Q_{2}, Q_{-1}\right]=-\frac{1}{2} J_{+},} & {\left[Q_{0}, Q_{1}\right]=-\frac{\sqrt{3}}{2 \sqrt{2}} J_{+},}
\end{array}
$$


together with their hermitian conjugates. The algebras (28), (30), and (31) are all nicely graded. However, to obtain a solution to the cubic algebra different from any of those preceding we must break the graded structure.

If we let

$$
\alpha_{ \pm}=J_{ \pm}, \quad \alpha_{0}=\lambda J_{0}+\mu\left(Q_{2}+Q_{-2}\right),
$$

with $\lambda, \mu$ real, then we find Eqs. (19) are satisfied provided

$$
\lambda^{2}+\mu^{2}=1 \text {. }
$$

To check $\lambda$, $\mu$ are not merely $\mathrm{SO}(3) \times \mathrm{SU}(3)$ parameters we compute the invariants $(q, t)$ and find,

$$
(q, t)=(6,6 \lambda),
$$

indicating a whole range of solutions for $|\lambda| \leqq 1$, running between the maximal embedding $(\lambda=1)$ and its anti-dual counterpart $(\lambda=-1)$.

The action of the commutator mapping on these solutions is interesting. Suppose we let

$$
\lambda=\cos \theta
$$

and compute $\tilde{\alpha}$. We find

$$
\tilde{\alpha}_{0}=J_{0}, \quad \tilde{\alpha}_{ \pm}=\lambda J_{ \pm} \mp 2 \mu Q_{\mp 1}=U(-\theta) J_{ \pm} U(\theta),
$$

where $U(\theta)$ is given by

$$
U(\theta)=\exp \theta\left(Q_{2}-Q_{-2}\right) .
$$

Thus, we can transform $\tilde{\alpha}_{ \pm}$to $J_{ \pm}$by an $\mathrm{SU}(3)$ transformation, and $\tilde{\alpha}_{0}$ becomes

$$
\tilde{\alpha}_{0}=U(\theta) J_{0} U(-\theta)=\cos 2 \theta J_{0}-\sin 2 \theta\left(Q_{2}+Q_{-2}\right) .
$$

Comparing (38) with (32) we see that $\tilde{\alpha}(\theta)$ is SU(3) equivalent to $\alpha(-2 \theta)$. In other words, the commutator map generally yields a different solution within the same family. It is equivalent to the mapping

$$
\theta \rightarrow-2 \theta \bmod (2 \pi)
$$

as far as the parameter $\theta$ is concerned.

Notice, the mapping (39) has three fixed points $\theta=0,2 \pi / 3,4 \pi / 3$. The point $\theta=0$ corresponds to the maximal embedding itself but the other two correspond to values of $\theta$ for which the $\alpha$ algebra does not close but fails to do so by a conjugation. That is,

$$
\left[\alpha_{i}, \alpha_{j}\right]=i \varepsilon_{i j k} w \alpha_{k} w^{\dagger},
$$

where $w$ is a matrix, Eq. (37), whose eigenvalues are the three roots of unity.

Repeated applications of maps such as (39) have been much studied by those interested in chaotic behaviour. See, for example, [9] and references therein. The evolution of successive iterations depends sensitively on the starting point. Apart from the above-mentioned fixed points there are cycles of any period as well as very erratic orbits whose evolution is essentially random. Whether these facts have any relevance in the present context is not known, however. 
Besides the SU(2) embeddings and their associated singular solutions, and the continuous parameter set, we have come across one more isolated solution. Given in terms of the maximal embedding Eqs. (28)-(31), an expression for it is

$$
\alpha_{+}=\frac{1}{\sqrt{3}} J_{+}=\left(\alpha_{-}\right)^{\dagger}, \quad \alpha_{0}=\frac{\sqrt{10}}{3} Q_{0} .
$$

For this solution the two invariants are calculated to be

$$
(q, t)=\left(\frac{22}{9}, 0\right) .
$$

It is the only solution, apart from the embeddings and singular ones, which has a symmetry; in this case a $\mathrm{U}(1)$ subgroup of $\mathrm{SO}(3) \times \mathrm{SU}(3)$. It also has a distinguishing feature with regard to the commutator map. For all the other solutions at least a double application of the map yielded a new solution. However, (42) is rather different. Starting with it repeated applications of the commutator map lead eventually (after an infinite number of steps) to zero, without encountering any other solutions on the way. Thus, incidentally, providing a counter-example to the at first plausible hypothesis that a finite number of applications of the map might produce a new solution from an old one.

The question of whether these solutions are the complete set in three dimensions is not yet answered, as such evidence as there is inconclusive. To study the solutions further we may regard them as stationary points of a particular functional and investigate the nature of these stationary points.

The cubic algebra is the condition for the stationary points of the function

$$
S=\operatorname{tr} \alpha^{2}+\frac{1}{4} \operatorname{tr}\left\{\left[\alpha_{i}, \alpha_{j}\right]\left[\alpha_{i}, \alpha_{j}\right]\right\} .
$$

$S$ is not positive, but at a stationary point it takes the value $\frac{1}{2} \operatorname{tr} \alpha^{2}$ which is certainly positive, vanishing only when $\alpha$ vanishes. Zero is a local minimum of $S$ but the character of the other stationary points is not easy to compute since it depends on knowledge of the eigenvalues of the quadratic fluctuation matrix, $M$, given by

$$
\begin{aligned}
M_{i l m, j p q}= & 2 \delta_{i j} \delta_{l p} \delta_{q m}-\delta_{i j}\left[\alpha_{q m}^{2} \delta_{l p}+\alpha_{l p}^{2} \delta_{q m}\right] \\
& -\left(\alpha_{i}\right)_{q m}\left(\alpha_{j}\right)_{l p}-\left(\alpha_{i}\right)_{l p}\left(\alpha_{j}\right)_{q m} \\
& +2\left(\alpha_{k}\right)_{q m}\left(\alpha_{k}\right)_{l p} \delta_{i j}+2\left(\alpha_{i} \alpha_{j}\right)_{q m} \delta_{l p} \\
& +2\left(\alpha_{j} \alpha_{i}\right)_{l p} \delta_{q m}-\left(\alpha_{i} \alpha_{j}\right)_{l p} \delta_{q m} \\
& -\left(\alpha_{j} \alpha_{i}\right)_{q m} \delta_{l p} .
\end{aligned}
$$

It is worth remarking that this matrix also appears in a different context. Suppose we set out to solve Eqs. (3), (4) for $T_{i}(z)$. We might begin by writing a formal power series

$$
T_{i}(z)=\frac{\alpha_{i}}{z+\frac{1}{2}}+\beta_{i}\left(z+\frac{1}{2}\right)^{\gamma}+\ldots
$$

near a pole. In which case, besides the cubic algebra for $\alpha$ we would find

$$
\begin{gathered}
\gamma(\gamma-1) \beta_{i}=\left[\alpha_{j},\left[\alpha_{j}, \beta_{i}\right]\right]+\left[\alpha_{j},\left[\beta_{j}, \alpha_{i}\right]\right]+\left[\beta_{j},\left[\alpha_{j}, \alpha_{i}\right]\right], \\
(\gamma+1)\left[\alpha_{j}, \beta_{j}\right]=0 .
\end{gathered}
$$


Equation (46) can be recast in terms of the fluctuation matrix $M$ to read

$$
M_{i j} \beta_{j}=\{2-\gamma(\gamma-1)\} \beta_{i},
$$

and the eigenvalues of $M$ determine the possible choices of $\gamma$. Only $\gamma$ being a positive integer or zero is acceptable in the sense that we are interested in solutions which are analytic apart from two poles at $z= \pm \frac{1}{2}$. Thus, only certain eigenvalues and the corresponding eigenvectors $\beta_{i}$ are acceptable, namely $2,0,-4, \ldots$, corresponding, respectively, to $\gamma=0,1,2,3, \ldots$. The solution represented by Eqs. (9), (12) corresponds to $\gamma=3$, in fact, with eigenvector $\alpha_{i}$ itself, incidentally demonstrating that no solution to the cubic algebra apart from zero is a local minimum of $S$, Eq. (43). The problem of guaranteeing the existence of a further pole at $z=\frac{1}{2}$ is very difficult, however.

Returning to the nature of the solutions to the cubic algebra, the least we ought to be able to do is discover the number of degrees of freedom of each solution, or, equivalently, to count the number of zero eigenvalues of $M$, in each case. For the maximal embedding the computation can be done algebraically and it turns out to have fifteen zero modes. Eight of these are the group freedom [taking into account the $\mathrm{SO}(3)$ invariance of the $\mathrm{SU}(3)$ embedding] but the other seven appear to be extra degrees of freedom. We might guess that four of these at least are involved in the deformation into the one parameter solution described above, Eq. (32), [the $\mathrm{SO}(3)$ plus one more parameter]. That this is indeed the case is also suggested numerically since the one parameter set has precisely twelve degrees of freedom $[\mathrm{SO}(3) \times \mathrm{SU}(3)$ plus a single parameter], at least at each of the points we have examined carefully. So, there might appear to be three extra degrees of freedom remaining. However, with respect to the $\mathrm{SO}(3)$ group under whose action the maximal embedding is invariant the whole set of seven degrees of freedom orthogonal to the group freedom are a septet (rather than a quartet and a triplet, or two triplets and a singlet both of which are impossible here). This suggests that the situation is actually rather like the vertex of a cone (say $z^{2}=x^{2}+y^{2}$ ). At the origin, a formal calculation suggests three degrees of freedom although we know the cone has just two. The maximal embedding has a symmetry which is clearly broken by neighbouring solutions, just like the cone. Thus we may regard the apparent seven degrees of freedom as the "ambient" space into which the four dimensional "cone" of solutions fits. There could be several more such cones disconnected from each other, but we have not in fact been able to find any. Indeed, we suspect there are none.

$N>3$. Here the issue is very complicated because of the variety of embeddings possible, both of SU(2) and the other sorts of solution described above. However, we should like to confine ourselves merely to a couple of remarks concerning the maximal embedding of $\mathrm{SU}(2)$ into $\mathrm{SU}(N)$. At first sight one might think that the maximal embedding solution to the cubic algebra would have more degrees of freedom in a larger group than SU(3). However, that is not the case, as the argument sketched in the appendix shows. In fact, the number of degrees of freedom is always exactly seven more than the dimension of $\mathrm{SU}(N)$, just as it was for $\mathrm{SU}(3)$. The maximal embedding organizes the generators of $\mathrm{SU}(N)$ into multiplets, one of dimension 3 (the embedding), and one each of dimension $5,7, \ldots, 2 N-1$. However, it is always the quintuplet that has the extra freedom to 
allow other solutions connected, but not equivalent, to the embedding itself. The rest correspond only to $\mathrm{SU}(N)$ transformations.

It is worth making the following parenthetical remark.

The distinguished role of the quintuplet in this context means that if we tried to restrict our $\alpha$ 's to be linear combinations of hermitian generators to groups other than $\mathrm{SU}(N)$ there would be no chance of finding any solution at all connected to the maximal embedding. It is easy to see, examining the Dynkin diagrams, that it is only for $\mathrm{SU}(N)$ that the maximal embedding organizes the generators to include a quintuplet. Remarkably, this phenomenon occurs for no other simple Lie algebra.

Having said that we have so far failed to find a set of solutions for $N>3$ connected to the maximal embedding, as we managed to do in the case $N=3$, Eq. (32).

\section{The Dirac Equation}

In this section we shall make a few preliminary remarks about the Dirac equation, but our analysis is far from complete.

In order to construct the self (anti) dual BPS monopoles via Nahm's construction it is necessary to solve the one dimensional left (right) handed Dirac equation [3]

$$
\left[\frac{d}{d z}(\overline{+}) \boldsymbol{\sigma} \cdot(\mathbf{x}+\mathbf{T}(z))\right] v(z)=0
$$

on the interval $|z| \leqq \frac{1}{2}$, picking out a complete set of normalizable solutions with respect to the inner product

$$
(v, v)=\int_{-1 / 2}^{1 / 2} d z v^{\dagger} v
$$

Here, $\mathbf{T}(z)$ satisfies (5) (taking the + sign) and has a simple pole at $z= \pm \frac{1}{2}$ with a residue which represents the $\mathrm{SU}(2)$ algebra irreducibly. Under these circumstances the left (right) handed part of Eq. (49) has precisely two normalizable solutions on the interval, whilst the right (left) handed part has none. The final step to construct the static SU(2) gauge field $A_{i}$, and Higgs field $\Phi$ in three dimensions uses these solutions organized into a single matrix

$$
v=\left(v_{1}, v_{2}\right)
$$

via the transformation

$$
\begin{gathered}
\Phi=\int_{-1 / 2}^{1 / 2} d z v^{\dagger} z v, \\
\mathbf{A}=\int_{-1 / 2}^{1 / 2} d z v^{\dagger}(-i \nabla v),
\end{gathered}
$$

where $v$ is assumed to be normalized:

$$
\mathbb{1}=\int_{-1 / 2}^{1 / 2} d z v^{\dagger} v
$$


It is then guaranteed that $\Phi, A_{i}$ satisfy the Bogomol'nyi equations and represent a monopole with magnetic charge $N$ [if $\mathbf{T}$ is an $S U(N)$ gauge field], and with a finite energy $4 \pi N$ in suitably chosen units.

When $\mathbf{T}(x)$ satisfies the second order equations (3), (4) instead of the self dual equations (5), both the left and right handed Dirac equations will be expected to have normalizable solutions [which is also the case when $\mathbf{T}(z)$ is a constant triple of three numbers a, corresponding via the above transformation to a single monopole (or antimonopole), depending on which choice of Dirac equation is selected to make the transformation]. There is no nice vanishing theorem as in the self-dual case when the right-handed equation has no normalizable solutions at all (for $N \geqq 2$ ). The question is whether there is a choice of $\mathbf{T}(z)$ leading via the above transformation to a solution to the second order SU(2) Yang-Mills-Higgs equations in the BPS limit.

Not much is known about the behaviour of solutions to the Dirac equation in a non-dual background and we are not able to solve Eq. (49) in general.

Here we shall confine ourselves to a discussion of some consequences of the poles in $\mathbf{T}(x)$ at $z= \pm \frac{1}{2}$. If the residue of a pole represents the $\mathrm{SU}(2)$ algebra, then it is easy to see (as Nahm pointed out), since $\sigma \cdot \alpha$ is then a "spin-orbit" coupling, that the solutions of Eq. (49) near the pole fall into two classes corresponding to the two eigenvalues of $\boldsymbol{\sigma} \cdot \boldsymbol{\alpha}$. That is,

$$
v_{i}(z) \sim\left(\frac{1}{2} \pm z\right)^{\lambda_{i}} V_{0 i}, \quad i=1,2,
$$

where, if we denote the spin of the irreducible $(N \times N)$ representation $\boldsymbol{\alpha}$ of SU(2) by $J_{\alpha}$,

$$
\left(\lambda_{1}, \lambda_{2}\right)=\left(J_{\alpha},-J_{\alpha}-1\right)=\left(\frac{N-1}{2},-\frac{N+1}{2}\right)
$$

with the dimensions of the corresponding eigenspaces being

$$
\left(d_{1}, d_{2}\right)=\left(2 J_{\alpha}+2,2 J_{\alpha}\right)=(N+1, N-1),
$$

respectively. In other words, the effect of a single pole is to reduce the number of normalizable solutions from a possible $2 N$ to $N+1$ (for the left handed equation), or $N-1$ (for the right handed equation).

If $\mathbf{T}(z)$ is self dual we also know that the right handed equation has no normalizable solutions at all on $|z| \leqq \frac{1}{2}$ so the other pole must be neatly arranged so that those $N-1$ solutions with a zero at one end all become singular at the other. This change over is reflected in $N-1$ of the left handed solutions, too, so just 2 of the $N+1$ can have zeros at both ends of the interval and hence be normalizable. This is, of course, precisely what is required to construct SU(2) gauge fields via the mapping $(52,53)$ and demonstrates that self duality together with the special pole residue is a powerful combination. For non-dual solutions there is no vanishing theorem and therefore no such strong principle. Nevertheless, we should like to point out the behaviour of a solution to Eq. (49) near a pole whose residue satisfies the cubic algebra. Indeed, for the case $N=3$ we have a whole set of pole residues interpolating self/anti-dual irreducible representations of the SU(2) algebra and we ought to be able to track the behaviour of the solutions as the parameter $\theta$ is varied. 
For general $\theta$ we find three eigenvalues of $\boldsymbol{\sigma} \cdot \boldsymbol{\alpha}$ each of multiplicity 2 . Thus, setting $\lambda=\sin \theta$, instead of (35) for convenience, the eigenvalues are

$$
\lambda_{k}=2 \sin \left(\frac{\theta}{3}+\frac{2 \pi k}{3}\right), \quad k=0,1,2 .
$$

For $\theta= \pm \frac{\pi}{2}$ the eigenvalues (multiplicities) are $\pm 1(4), \mp 2(2)$, respectively, in agreement with Eq. (56), (57). For $-\frac{\pi}{2}<\theta<\frac{\pi}{2}$ there are three distinct eigenvalues. Notice, the eigenvalues flow with $\theta$ in such a way that the quantity $v$ defined by

$$
\begin{array}{r}
v=\text { (number of nonsingular left hand solutions - } \\
\text { number of nonsingular right hand solutions) }
\end{array}
$$

jumps from 2 , in the range $0<\theta \leqq \frac{\pi}{2}$, to -2 in the range $-\frac{\pi}{2} \leqq \theta<0$. At $\theta=0 v$ vanishes. $\theta=0$ is a crossover point, where one pair of eigenvalues changes sign, the other pairs have the same sign over the whole interval. Some singular solutions are normalizable, however, and for these the corresponding $v$ jumps when $\sin \theta / 3=-\frac{1}{4}$. Thus, there is an interval in which there are only two normalizable left-handed solutions even for a single pole.

To date we have failed to find a convincing way of approaching a Taubes' non-dual monopole via this method. We have not been able to solve the various equations. Nahm's second order Eqs. (3) and linear Eq. (49), in particular, in enough detail to test the hypothesis. Nevertheless, we feel the cubic algebra by itself is an interesting problem that would repay further study and warrant the digression we have made. We were surprised at the complexity already present for $N=3$.

Many questions remain. With regard to the cubic algebra itself we should like a classification of all the possibilities, particularly, those that have extra degrees of freedom. At the moment we do not even have a convincing proof that for a given dimension the quadratic invariant $q$ is bounded on the set of solutions. The commutator map appears to divide the configuration space of the three matrices into two pieces, one for which all iterations eventually converge, the other for which they do not. We do not know the detailed nature of these two pieces. For the second order differential equations we should like to know the possible developments away from the poles, and which pole residues can occur in combination at $z= \pm \frac{1}{2}$. Finally, we need to know more about the Dirac equation (49), we have not found any case so far for which we can carry out the Nahm transformation to test the hypothesis.

\section{Appendix}

In this section we sketch a proof of the statement in Sect. 3 concerning the zero modes of the fluctuation matrix $M$, and the quintuplet of generators. We can argue directly from Eqs. (19), setting

$$
\alpha_{i}=J_{i}+\beta_{i}, \quad i= \pm 1,0,
$$

and satisfying the equations to first order in $\beta$. Since the maximal embedding organizes the generators into spin multiplets, one of each integer spin from 1 to $N-1$, we may write

$$
\beta_{i}=\sum_{j=1}^{N-1} \sum_{m=-j}^{j} b_{m}^{i}(j) T_{m}^{(j)}
$$


where the $T_{m}^{(j)}, j=1, \ldots, N-1,|m| \leqq j$ are the generators of $\mathrm{SU}(N)$. To first order in $\beta$ the Eqs. (19) will not mix spins so we may consider each $j$ separately in Eq. (A.2). In addition, for each $j$ the coefficients satisfy

$$
\left(b_{m}^{0}\right)^{*}=(-)^{m} b_{-m}^{0}, \quad\left(b_{m}^{+}\right)^{*}=(-)^{m} b_{-m}^{-}
$$

[with the conventional choice $T_{m}^{\dagger}=(-)^{m} T_{-m}$ ], to ensure the hermiticity of $\alpha_{i}$.

For each $j$, Eqs. (19) imply:

$$
\begin{gathered}
2\left(j(j+1)-\left(m^{2}+2\right)\right) b_{m}^{0}+(2-m) N_{m-1}^{+} b_{m-1}^{-}-(m+2) N_{m+1}^{-} b_{m+1}^{+}=0, \\
-(m+2) N_{m}^{+} b_{m}^{0}-\frac{1}{2} N_{m-1}^{+} N_{m}^{+} b_{m-1}^{-}+\frac{1}{2}(j(j+1)+m(m+5)) b_{m+1}^{+}=0,
\end{gathered}
$$

where the coefficients $N_{m}^{ \pm}$are defined by

$$
N_{m}^{ \pm}=[(j \pm m)(j \pm m+1)]^{1 / 2}
$$

If $j \neq 2$ we may solve Eqs. (A.4), (A.5) to find:

$$
\begin{gathered}
b_{m+1}^{+}=\left(\frac{(j-m)(j+m+1)}{(j+m)(j-m+1)}\right)^{1 / 2} b_{m-1}^{-}, \\
b_{m}^{0}=\frac{1}{2} \frac{(j(j+1)+3 m(m+3))}{(m+2) N_{m}^{-}} b_{m-1}^{-} .
\end{gathered}
$$

Hence, in this case each of $b_{m}^{ \pm}$is determined by the $b_{m}^{0}$ which, in view of the hermiticity requirements (A.3) carry $2 j+1$ parameters. These correspond to the freedom of $\mathrm{SU}(N)$ transformations generated by $T_{m}^{(j)}$.

If $j=2$ there are two cases $|m|=2,|m| \neq 2$. For $m= \pm 2(\mathrm{~A} .4)$ and (A.5) are identities. Otherwise, for $|m|<2$ we have a single relation

$$
b_{m}^{0}=\frac{1}{2}\left(\frac{3+m}{2-m}\right)^{1 / 2} b_{m+1}^{+}-\frac{1}{2}\left(\frac{3-m}{2+m}\right)^{1 / 2} b_{m-1}^{-} .
$$

Thus, for $j=2$ the available parameters are contained in $b_{m}^{ \pm},|m| \leqq 2$ and $b_{2}^{0}$, a total of 12 . Five of these are $\mathrm{SU}(N)$ transformations, but the other seven are the extra ones referred to in the text.

Acknowledgements. We should like to thank our colleagues for remarks and discussion, in particular Roland Warner. One of us (PRW) wishes to thank the U.K. Science and Engineering Research Council for a Studentship.

\section{References}

1. Baseyan, G.Z., Martinyan, S.G., Savviddy, G.K.: Non-linear plane waves in the massless YangMills theory. Pis'ma Zh. Gksp. Teor. Prakt. Fiz. Kul. 29, 641 (1979)

2. Nikolaevskii, E.S., Schur, L.N.: Non-integrability of the classical Yang-Mills fields. JETP Letts. 36, 218 (1982)

Savviddy, G.K.: The Yang-Mills classical mechanics as a Kolmogarov $K$-system. Phys. Letts. 130 B, 303 (1983)

Chang, S.J.: Classical Yang-Mills solutions and iterative maps. Phys. Rev. D 29, 259 (1984) 
3. Atiyah, M.F., Hitchin, N.J., Drinfeld, V.G., Manin, Yu.I.: Construction of instantons. Phys. Letts. B 65 A, 185 (1978)

Drinfeld, V.G., Manin, Yu.I.: A description of instantons. Commun. Math. Phys. 63, 177 (1978) Nahm, W.: Multimonopoles in the ADHM construction. In: Proceedings of the symposium on particle physics. Horvath, Z., et al. (eds.), Visegrad 1981

Nahm, W.: Construction of all self-dual monopoles by the ADHM method. In: Monopoles and quantum field theory. Craigie, N., et al. (eds.), Singapore: World Scientific 1982

Hitchin, N.J.: On the construction of monopoles. Commun. Math. Phys. 89, 145 (1983)

4. Bogoml'nyi, E.B.: The stability of classical solutions. Sov. J. Nucl. Phys. 24, 449 (1976)

Prasad, M.K., Sommerfield, C.M.: Exact classical solution for the 't Hooft monopole and JuliaZee dyon. Phys. Rev. Lett. 35, 760 (1975)

5. Corrigan, E.: A brief introduction to the monopole. Talk given at 11th winter school on abstract analysis. Zelezna Ruda, Czechoslovakia, Jan. 1983

Corrigan, E., Goddard, P.: Construction of instanton and monopole solutions and reciprocity. Ann. Phys. (N.Y.) 154, 352 (1984)

Nahm, W.: Self dual monopoles and calorons. Talk given at XII colloquium on group theoretical methods in physics. Trieste, September 1983

6. Taubes, C.: The existence of a non-minimal solution to the SU(2) Yang-Mills-Higgs equations on $\mathbb{R}^{3}$. Commun. Math. Phys. 86, 257 (1982)

7. Erdelyi, A., et al.: Higher transcendental functions, Vol. II. New York: McGraw-Hill 1953

8. Wybourne, B.G.: Classical groups for physicists. New York: Wiley 1974

9. Cvitanovic, P., Myrheim, J.: Complex universality. Nordita preprint $84 / 5$

Manton, N.S., Nauenberg, M.: Universal scaling behaviour for iterated maps in the complex plane. Commun. Math. Phys. 89, 555 (1983)

Communicated by A. Jaffe

Received August 22, 1984 